Special Issue: 25 years of the Mizar Mathematical Library

FORMALIZED MATHEMATICS

Vol. 22, No. 2, Pages $157-166, \mathbf{2 0 1 4}$

DOI: 10.2478 /forma-2014-0016

\title{
Cauchy Mean Theorem
}

\author{
Adam Grabowski \\ Institute of Informatics \\ University of Białystok \\ Akademicka 2, 15-267 Białystok \\ Poland
}

\begin{abstract}
Summary. The purpose of this paper was to prove formally, using the Mizar language, Arithmetic Mean/Geometric Mean theorem known maybe better under the name of AM-GM inequality or Cauchy mean theorem. It states that the arithmetic mean of a list of a non-negative real numbers is greater than or equal to the geometric mean of the same list.

The formalization was tempting for at least two reasons: one of them, perhaps the strongest, was that the proof of this theorem seemed to be relatively easy to formalize (e.g. the weaker variant of this was proven in [13]). Also Jensen's inequality is already present in the Mizar Mathematical Library. We were impressed by the beauty and elegance of the simple proof by induction and so we decided to follow this specific way.

The proof follows similar lines as that written in Isabelle [18; the comparison of both could be really interesting as it seems that in both systems the number of lines needed to prove this are really close.

This theorem is item \#38 from the "Formalizing 100 Theorems" list maintained by Freek Wiedijk at http://www.cs.ru.nl/F.Wiedijk/100/.
\end{abstract}

MSC: 26A06 26A12 03B35

Keywords: geometric mean; arithmetic mean; AM-GM inequality; Cauchy mean theorem

MML identifier: RVSUM_3, version: 8.1.03 5.23.1210

The notation and terminology used in this paper have been introduced in the following articles: [1, [2], 7], [5], [12, [20, [9], 6], 23], 21], [3, [17], 4], 15], [19], 14], 26], [16], 10], 22], 25], and [11]. 


\section{Preliminaries}

Let us consider real numbers $x, y, z, w$. Now we state the propositions:

(1) If $|x-y|<|z-w|$, then $(x-y)^{2}<(z-w)^{2}$.

(2) If $|x-y|<|z-w|$ and $x+y=z+w$, then $x \cdot y>z \cdot w$. The theorem is a consequence of (1).

Let $f$ be a real-valued finite sequence. We introduce $f$ is positive as a synonym of $f$ is positive yielding.

Observe that $f$ is positive if and only if the condition (Def. 1) is satisfied.

(Def. 1) Let us consider a natural number $n$. If $n \in \operatorname{dom} f$, then $f(n)>0$.

Note that there exists a real-valued finite sequence which is non empty, constant, and positive and there exists a real-valued finite sequence which is non empty, non constant, and positive.

Let $f$ be a non empty real-valued finite sequence and $n$ be a natural number. One can verify that $f\lceil\operatorname{Seg} n$ is real-valued.

Let $f$ be a positive non empty real-valued finite sequence. Let us note that $f\lceil\operatorname{Seg} n$ is positive.

Let $f$ be a finite sequence. We introduce $f$ is homogeneous as a synonym of $f$ is constant.

Let $f$ be a finite sequence. We introduce $f$ is heterogeneous as an antonym of $f$ is homogeneous.

Let us consider real-valued finite sequences $R_{1}, R_{2}$. Now we state the propositions:

(3) Suppose len $R_{1}=$ len $R_{2}$ and for every natural number $j$ such that $j \in$ Seg len $R_{1}$ holds $R_{1}(j) \leqslant R_{2}(j)$ and there exists a natural number $j$ such that $j \in$ Seg len $R_{1}$ and $R_{1}(j)<R_{2}(j)$. Then $\sum R_{1}<\sum R_{2}$.

(4) If $R_{1}$ and $R_{2}$ are fiberwise equipotent, then $\prod R_{1}=\prod R_{2}$. Proof: Define $\mathcal{P}$ [natural number] $\equiv$ for every finite sequences $f, g$ of elements of $\mathbb{R}$ such that $f$ and $g$ are fiberwise equipotent and len $f=\$_{1}$ holds $\prod f=\prod g$. For every natural number $n$ such that $\mathcal{P}[n]$ holds $\mathcal{P}[n+1]$ by [2, (75)], [3, (13)], [24, (25)], [8, (10), (4), (5)]. $\mathcal{P}[0]$ by [16, (3)]. For every natural number $n, \mathcal{P}[n]$ from [3, Sch. 2].

\section{Arithmetic Mean and Geometric Mean}

Let $f$ be a real-valued finite sequence. The functor Mean $f$ yielding a real number is defined by the term

(Def. 2) $\quad \frac{\sum f}{\operatorname{len} f}$.

Let $f$ be a positive real-valued finite sequence. The functor GMean $f$ yielding a real number is defined by the term 
(Def. 3) $\quad \sqrt[\operatorname{len} f]{\prod f}$.

Let us consider a real-valued finite sequence $f$. Now we state the propositions:

(5) $\sum f=\operatorname{len} f \cdot \operatorname{Mean} f$.

(6) $\operatorname{Mean}\left(f^{\frown}\langle\right.$ Mean $\left.f\rangle\right)=\operatorname{Mean} f$. The theorem is a consequence of (5).

Let $f$ be a non empty constant real-valued finite sequence. Observe that the value of $f$ is real.

Let us consider a non empty constant real-valued finite sequence $f$. Now we state the propositions:

(7) $\sum f=($ the value of $f) \cdot \operatorname{len} f$.

(8) $\prod f=(\text { the value of } f)^{\operatorname{len} f}$.

(9) Mean $f=$ the value of $f$. The theorem is a consequence of (7).

Let us consider a non empty constant positive real-valued finite sequence $f$. Now we state the propositions:

(10) The value of $f>0$.

(11) GMean $f=$ the value of $f$. The theorem is a consequence of (10) and (8).

Let $f$ be a non empty positive real-valued finite sequence. Observe that Mean $f$ is positive.

Let us note that $\prod f$ is positive.

Let $f$ be a positive non empty real-valued finite sequence. Note that GMean $f$ is positive.

\section{Heterogeneity of a Finite Sequence}

Let $f$ be a real-valued finite sequence. The functor HetSet $f$ yielding a subset of $\mathbb{N}$ is defined by the term

(Def. 4) $\{n$, where $n$ is a natural number : $n \in \operatorname{dom} f$ and $f(n) \neq$ Mean $f\}$.

One can verify that HetSet $f$ is finite.

Let $f$ be a positive non empty real-valued finite sequence. Let us observe that HetSet $f$ is upper bounded lower bounded and real-membered.

Let $f$ be a real-valued finite sequence. The functor Het $f$ yielding a natural number is defined by the term

(Def. 5) $\overline{\overline{\operatorname{HetSet} f}}$.

Now we state the propositions:

(12) Let us consider a real-valued finite sequence $f$. If Het $f=0$, then $f$ is homogeneous.

(13) Let us consider a non empty real-valued finite sequence $f$. If Het $f \neq 0$, then $f$ is heterogeneous. The theorem is a consequence of (9). 
Let $f$ be a heterogeneous positive non empty real-valued finite sequence. Note that HetSet $f$ is non empty.

Now we state the proposition:

(14) Let us consider a non empty homogeneous positive real-valued finite sequence $f$. Then Mean $f=$ GMean $f$. The theorem is a consequence of (9) and (11).

Let $f_{1}, f_{2}$ be real-valued finite sequences. We say that $f_{1}$ and $f_{2}$ are $\gamma$ equivalent if and only if

(Def. 6) (i) len $f_{1}=\operatorname{len} f_{2}$, and

(ii) Mean $f_{1}=$ Mean $f_{2}$.

One can check that the predicate is reflexive and symmetric.

Now we state the proposition:

(15) Let us consider real-valued finite sequences $f_{1}, f_{2}$. Suppose

(i) $\operatorname{dom} f_{1}=\operatorname{dom} f_{2}$, and

(ii) $\sum f_{1}=\sum f_{2}$.

Then $f_{1}$ and $f_{2}$ are $\gamma$-equivalent.

Let $f$ be a real-valued finite sequence. The functors: MeanLess $f$ and

MeanMore $f$ yielding subsets of $\mathbb{N}$ are defined by terms,

(Def. 7) $\{n$, where $n$ is a natural number $: n \in \operatorname{dom} f$ and $f(n)<$ Mean $f\}$,

(Def. 8) $\{n$, where $n$ is a natural number : $n \in \operatorname{dom} f$ and $f(n)>$ Mean $f\}$, respectively.

Let us consider a real-valued finite sequence $f$. Now we state the propositions:

(16) $\operatorname{HetSet} f \subseteq \operatorname{dom} f$.

(17) MeanLess $f \subseteq \operatorname{dom} f$.

(18) MeanMore $f \subseteq \operatorname{dom} f$.

(19) HetSet $f=$ MeanLess $f \cup$ MeanMore $f$.

Let $f$ be a heterogeneous real-valued finite sequence. One can verify that MeanLess $f$ is non empty and MeanMore $f$ is non empty.

Let $f$ be a homogeneous real-valued finite sequence.

Let us note that MeanLess $f$ is empty and MeanMore $f$ is empty.

Let us consider a heterogeneous non empty real-valued finite sequence $f$. Now we state the propositions:

(20) MeanLess $f$ misses MeanMore $f$.

(21) Het $f \geqslant 2$. The theorem is a consequence of (19) and (20). 


\section{Auxiliary Replacement Function}

Let $f$ be a function, $i, j$ be natural numbers, and $a, b$ be objects. The functor $\operatorname{Replace}(f, i, j, a, b)$ yielding a function is defined by the term

(Def. 9) $(f+\cdot(i, a))+\cdot(j, b)$.

Now we state the proposition:

(22) Let us consider a finite sequence $f$, natural numbers $i, j$, and objects $a$, $b$. Then $\operatorname{dom} \operatorname{Replace}(f, i, j, a, b)=\operatorname{dom} f$.

Let $f$ be a real-valued finite sequence, $i, j$ be natural numbers, and $a, b$ be real numbers. Let us observe that $\operatorname{Replace}(f, i, j, a, b)$ is real-valued and finite sequence-like.

Now we state the propositions:

(23) Let us consider a real-valued finite sequence $w$, a real number $r$, and a natural number $i$. Suppose $i \in \operatorname{dom} w$. Then $w+\cdot(i, r)=\left(\left(w\left\lceil\left(i--^{\prime} 1\right)\right)^{\frown}\right.\right.$ $\langle r\rangle)^{\frown} w_{\mid i}$.

(24) Let us consider a real-valued finite sequence $f$, a natural number $i$, and a real number $a$. If $i \in \operatorname{dom} f$, then $\sum(f+\cdot(i, a))=\sum f-f(i)+a$. The theorem is a consequence of (23).

(25) Let us consider a positive real-valued finite sequence $f$, a natural number $i$, and a real number $a$. Suppose $i \in \operatorname{dom} f$. Then $\prod(f+\cdot(i, a))=\frac{\prod f \cdot a}{f(i)}$. The theorem is a consequence of (23).

(26) Let us consider a real-valued finite sequence $f$, natural numbers $i, j$, and real numbers $a, b$. Suppose

(i) $i, j \in \operatorname{dom} f$, and

(ii) $i \neq j$.

Then $\sum$ Replace $(f, i, j, a, b)=\sum f-f(i)-f(j)+a+b$. The theorem is a consequence of (24).

(27) Let us consider a positive real-valued finite sequence $f$, natural numbers $i, j$, and positive real numbers $a, b$. Suppose

(i) $i, j \in \operatorname{dom} f$, and

(ii) $i \neq j$.

Then $\prod \operatorname{Replace}(f, i, j, a, b)=\frac{\prod f \cdot a \cdot b}{f(i) \cdot f(j)}$. PRoOF: For every natural number $n$ such that $n \in \operatorname{dom}(f+\cdot(i, a))$ holds $(f+\cdot(i, a))(n)>0$ by [6, (30), (31), $(32)]$. ПReplace $(f, i, j, a, b)=\frac{\prod(f+\cdot(i, a)) \cdot b}{(f+\cdot(i, a))(j)}$.

(28) Let us consider a real-valued finite sequence $f$ and natural numbers $i, j$. Suppose

(i) $i, j \in \operatorname{dom} f$, and 
(ii) $i \neq j$.

Then $f$ and Replace $(f, i, j$, Mean $f,(f(i)+f(j)$-Mean $f))$ are $\gamma$-equivalent. The theorem is a consequence of $(22)$ and (26).

(29) Let us consider a real-valued finite sequence $f$, natural numbers $i, j, k$, and real numbers $a, b$. Suppose

(i) $i, j, k \in \operatorname{dom} f$, and

(ii) $i \neq j$, and

(iii) $k \neq i$, and

(iv) $k \neq j$.

Then $(\operatorname{Replace}(f, i, j, a, b))(k)=f(k)$.

Let us consider a finite sequence $f$, natural numbers $i, j$, and objects $a, b$.

Let us assume that $i, j \in \operatorname{dom} f$ and $i \neq j$. Now we state the propositions:

(30) $\quad($ Replace $(f, i, j, a, b))(j)=b$.

(31) $\quad($ Replace $(f, i, j, a, b))(i)=a$.

Now we state the propositions:

(32) Let us consider a real-valued finite sequence $f$ and natural numbers $i, j$. Suppose

(i) $i, j \in \operatorname{dom} f$, and

(ii) $i \neq j$, and

(iii) $f(i) \neq$ Mean $f$, and

(iv) $f(j) \neq$ Mean $f$.

Then Het $f>\operatorname{Het} \operatorname{Replace}(f, i, j$, Mean $f,(f(i)+f(j)-$ Mean $f))$. The theorem is a consequence of (28), (31), (22), and (29).

(33) Let us consider positive non empty real-valued finite sequences $f, g$. Suppose

(i) len $f=\operatorname{len} g$, and

(ii) $\prod f<\prod g$.

Then GMean $f<$ GMean $g$.

(34) Let us consider a positive heterogeneous non empty real-valued finite sequence $f$. Then there exist natural numbers $i, j$ such that

(i) $i, j \in \operatorname{dom} f$, and

(ii) $i \neq j$, and

(iii) $f(i)<$ Mean $f<f(j)$.

Let us consider a positive heterogeneous non empty real-valued finite sequence $f$ and natural numbers $i, j$. Now we state the propositions: 
(35) If $i, j \in \operatorname{dom} f$ and $i \neq j$ and $f(i)>\operatorname{Mean} f$, then Replace $(f, i, j$, Mean $f$, $(f(i)+f(j)-$ Mean $f))$ is positive. The theorem is a consequence of $(22)$, (31), (30), and (29).

(36) If $i, j \in \operatorname{dom} f$ and $i \neq j$ and $f(j)>$ Mean $f$, then Replace $(f, i, j$, Mean $f$, $(f(i)+f(j)-$ Mean $f))$ is positive. The theorem is a consequence of $(22)$, (31), (30), and (29).

Now we state the propositions:

(37) Let us consider a positive heterogeneous non empty real-valued finite sequence $f$. Then there exist natural numbers $i, j$ such that

(i) $i, j \in \operatorname{dom} f$, and

(ii) $i \neq j$, and

(iii) there exists a positive non empty real-valued finite sequence $g$ such that $g=\operatorname{Replace}(f, i, j$, Mean $f,(f(i)+f(j)-$ Mean $f))$ and GMean $f<$ GMean $g$.

The theorem is a consequence of (34), (22), (35), (27), and (33).

(38) Let us consider a heterogeneous non empty real-valued finite sequence $f$ and natural numbers $i, j$. Suppose

(i) $i=$ the element of MeanLess $f$, and

(ii) $j=$ the element of MeanMore $f$.

Then

(iii) $i, j \in \operatorname{dom} f$, and

(iv) $i \neq j$, and

(v) $f(i)<$ Mean $f$, and

(vi) $f(j)>$ Mean $f$.

(39) Let us consider a heterogeneous positive non empty real-valued finite sequence $f$ and objects $i, j$. Suppose

(i) $i \in$ MeanLess $f$, and

(ii) $j \in$ MeanMore $f$.

Then

(iii) $i, j \in \operatorname{dom} f$, and

(iv) $i \neq j$, and

(v) $f(i)<\operatorname{Mean} f$, and

(vi) $f(j)>$ Mean $f$.

Let us consider a positive heterogeneous non empty real-valued finite sequence $f$ and natural numbers $i, j$. Now we state the propositions: 
(40) Suppose $i, j \in \operatorname{dom} f$ and $i \neq j$ and $i \in$ MeanMore $f$ and $j \in$ MeanLess $f$. Then there exists a positive non empty real-valued finite sequence $g$ such that

(i) $g=\operatorname{Replace}(f, i, j$, Mean $f,(f(i)+f(j)-$ Mean $f))$, and

(ii) GMean $f<$ GMean $g$.

The theorem is a consequence of (39), (22), (35), (27), and (33).

(41) Suppose $i, j \in \operatorname{dom} f$ and $i \neq j$ and $j \in$ MeanMore $f$ and $i \in$ MeanLess $f$. Then there exists a positive non empty real-valued finite sequence $g$ such that

(i) $g=\operatorname{Replace}(f, i, j$, Mean $f,(f(i)+f(j)-$ Mean $f))$, and

(ii) GMean $f<$ GMean $g$.

The theorem is a consequence of (39), (22), (36), (27), and (33).

\section{Homogenization of a Finite Sequence}

Let $f$ be a heterogeneous positive non empty real-valued finite sequence. The functor Homogen $f$ yielding a real-valued finite sequence is defined by

(Def. 10) There exist natural numbers $i, j$ such that

(i) $i=$ the element of MeanLess $f$, and

(ii) $j=$ the element of MeanMore $f$, and

(iii) $i t=\operatorname{Replace}(f, i, j$, Mean $f,(f(i)+f(j)-$ Mean $f))$.

Now we state the proposition:

(42) Let us consider a heterogeneous positive non empty real-valued finite sequence $f$. Then $\operatorname{dom}$ Homogen $f=\operatorname{dom} f$. The theorem is a consequence of $(22)$.

Let $f$ be a heterogeneous positive non empty real-valued finite sequence. Note that Homogen $f$ is non empty.

Observe that Homogen $f$ is positive.

Let us consider a heterogeneous positive non empty real-valued finite sequence $f$. Now we state the propositions:

(43) Het Homogen $f<\operatorname{Het} f$. The theorem is a consequence of (38) and (32).

(44) Homogen $f$ and $f$ are $\gamma$-equivalent. The theorem is a consequence of (38) and (28).

(45) GMean Homogen $f>$ GMean $f$. The theorem is a consequence of (39) and (41). 


\section{Cauchy Mean Theorem}

Now we state the proposition:

(46) Let us consider a heterogeneous positive non empty real-valued finite sequence $f$. Then there exists a non empty homogeneous positive realvalued finite sequence $g$ such that

(i) GMean $g>$ GMean $f$, and

(ii) Mean $g=$ Mean $f$.

Proof: Define $\mathcal{P}$ [natural number] $\equiv$ there exists a positive non empty real-valued finite sequence $g$ such that Het $g=\$_{1}$ and Mean $f=$ Mean $g$ and GMean $g>$ GMean $f$ and Het $g<\operatorname{Het} f$. There exists a natural number $k$ such that $\mathcal{P}[k]$. For every natural number $k$ such that $k \neq 0$ and $\mathcal{P}[k]$ there exists a natural number $n$ such that $n<k$ and $\mathcal{P}[n] . \mathcal{P}[0]$ from [3, Sch. 7].

Now we state the proposition:

(47) Inequality of ARITHMEtic AND GEOMETRIC MEANs:

Let us consider a non empty positive real-valued finite sequence $f$. Then GMean $f \leqslant$ Mean $f$. The theorem is a consequence of (14), (13), and (46).

\section{REFERENCES}

[1] Grzegorz Bancerek. Cardinal numbers. Formalized Mathematics, 1(2):377-382, 1990.

[2] Grzegorz Bancerek. Tarski's classes and ranks. Formalized Mathematics, 1(3):563-567, 1990.

[3] Grzegorz Bancerek. The fundamental properties of natural numbers. Formalized Mathematics, 1(1):41-46, 1990.

[4] Grzegorz Bancerek. The ordinal numbers Formalized Mathematics, 1(1):91-96, 1990.

[5] Grzegorz Bancerek and Krzysztof Hryniewiecki. Segments of natural numbers and finite sequences Formalized Mathematics, 1(1):107-114, 1990.

[6] Grzegorz Bancerek and Andrzej Trybulec. Miscellaneous facts about functions. Formalized Mathematics, 5(4):485-492, 1996.

[7] Czesław Byliński. The complex numbers Formalized Mathematics, 1(3):507-513, 1990.

[8] Czesław Byliński. Finite sequences and tuples of elements of a non-empty sets Formalized Mathematics, 1(3):529-536, 1990.

[9] Czesław Byliński. Functions and their basic properties Formalized Mathematics, 1(1): 55-65, 1990

[10] Czesław Byliński. The sum and product of finite sequences of real numbers. Formalized Mathematics, 1(4):661-668, 1990.

[11] Czesław Byliński. Some basic properties of sets. Formalized Mathematics, 1(1):47-53, 1990.

[12] Agata Darmochwał. Finite sets Formalized Mathematics, 1(1):165-167, 1990.

[13] Fuguo Ge and Xiquan Liang. On the partial product of series and related basic inequalities. Formalized Mathematics, 13(3):413-416, 2005.

[14] Andrzej Kondracki. Basic properties of rational numbers Formalized Mathematics, 1(5): 841-845, 1990.

[15] Artur Korniłowicz. On the real valued functions Formalized Mathematics, 13(1):181-187, 2005.

[16] Jarosław Kotowicz. Functions and finite sequences of real numbers Formalized Mathematics, 3(2):275-278, 1992. 
[17] Rafał Kwiatek. Factorial and Newton coefficients. Formalized Mathematics, 1(5):887-890, 1990.

[18] Benjamin Porter. Cauchy's mean theorem and the Cauchy-Schwarz inequality. Archive of Formal Proofs, March 2006. ISSN 2150-914x. http://afp.sf.net/entries/Cauchy . shtml, Formal proof development.

[19] Konrad Raczkowski and Andrzej Nędzusiak. Real exponents and logarithms Formalized Mathematics, 2(2):213-216, 1991.

[20] Andrzej Trybulec. Binary operations applied to functions Formalized Mathematics, 1 (2):329-334, 1990.

[21] Andrzej Trybulec. On the sets inhabited by numbers. Formalized Mathematics, 11(4): $341-347,2003$.

[22] Andrzej Trybulec and Czesław Byliński. Some properties of real numbers Formalized Mathematics, 1(3):445-449, 1990.

[23] Michał J. Trybulec. Integers Formalized Mathematics, 1(3):501-505, 1990.

[24] Wojciech A. Trybulec. Non-contiguous substrings and one-to-one finite sequences. Formalized Mathematics, 1(3):569-573, 1990.

[25] Zinaida Trybulec. Properties of subsets. Formalized Mathematics, 1(1):67-71, 1990.

[26] Edmund Woronowicz. Relations and their basic properties. Formalized Mathematics, 1 (1):73-83, 1990 .

Received June 13, 2014 